\title{
Opiniones y creencias de embarazadas en control prenatal relacionadas con salud oral y embarazo
}

\section{Opinion and beliefs of pregnancy women in prenatal control related to oral health and pregnant}

\author{
Fuentes Fernández R*, Oporto Venegas G*, Alarcón AM**, Bustos Medina L**, \\ Prieto Gómez $\mathrm{R}^{* * *}$, Rico Williams $\mathrm{H}^{* * *}$
}

\section{RESUMEN}

Los procesos vitales son motivo de preguntas para la humanidad. La cultura popular da respuestas a éstas, no siempre científicamente, transformando las creencias en mitos. En salud oral, se cree que las embarazadas desarrollarían caries más fácilmente que la población general, considerándose normal que "por cada hijo se pierda un diente", y mujeres embarazadas no desean recibir atención odontológica por el daño que podría producir al feto el uso de anestésicos locales, hecho importante ante la carencia de información del bajo riesgo que poseen para el paciente éstos procedimientos por algunos médicos, quienes rehusan someter a embarazadas a tratamientos dentales. La cultura tiene una profunda relación en el estilo de vida, sociabilización y autoayuda, mecanismos de intervención que serían cimientos que basan la promoción en salud.

La investigación busca conocer las ideas que posee un grupo de embarazadas en control prenatal, en torno a la salud oral y el embarazo.

Fue confeccionada una encuesta con 5 preguntas cerradas, aplicándose a 65 embarazadas seleccionadas aleatoriamente. La muestra fue obtenida por conveniencia.

El 98\% de las encuestadas cree que durante el embarazo es necesario consultar al odontólogo, el $60 \%$ cree que el embarazo produce caries y el $70 \%$ cree que el embarazo puede producir pérdida de piezas dentarias. Se requieren más esfuerzos en educación durante el control prenatal para entregar conocimientos adecuados a las embarazadas en relación a salud oral y embarazo, incluyendo el factor cultural, que les permita mejorar las medidas de auto cuidado durante este período.

Palabras Clave: Odontología preventiva, promoción en salud, relación materno-fetal.

Palabras clave: Odontología preventiva, promoción en salud, relación materno-fetal.

\section{SUMMARY}

Vital processes have created questions to humankind. People's culture gives non-scientific response to some of it, changing beliefs into myths. Related to oral health, several cultures believe that pregnant women could suffer more frequently dental caries than general population, is considered normal that "by each one baby, mother lost a teeth", and some pregnant patients are not able to receive dental treatment, because this procedures could produce damages to fetus in develop; important situation considering absence of information that dental

* Departamento de Odontología Integral. Facultad de Medicina Universidad de La Frontera. Temuco, Chile.

** Departamento de Salud Pública. Facultad de Medicina. Universidad de La Frontera. Temuco, Chile.

*** Carrera de Obstetricia y Puericultura. Facultad de Medicina. Universidad de La Frontera. Temuco, Chile. 
procedures expose to such a low risk to patients in some physicians, whom refuse authorize to pregnant women to receive dental treatment. Culture has a deep relationship to life styles, social relations and self-help; in these intervention mechanisms are based health promotion.

The research seeks to know the ideas have a group of pregnant women in prenatal control related to oral health and pregnant.

Was made a survey with 5 closed questions, applied to 65 pregnant woman randomly selected.

$98 \%$ of women think that through pregnant period is necessary to visit a dentist, $60 \%$ believe that pregnant cause dental caries and $70 \%$ thinks that pregnant could cause lost of tooth.

Mayor efforts in education, considering cultural factors, are required through prenatal control to improve knowledge as well as self care in oral health in these patients group..

Key words: Preventive dentistry, health promotion, maternal fetal relation.

Fecha de recepción: 4 de marzo 2009.

Aceptado para publicación: 12 de marzo 2009.

Fuentes Fernández R, Oporto Venegas G, Alarcón AM, Bustos Medina L, Prieto Gómez R, Rico Williams H. Opiniones y creencias de embarazadas en control prenatal relacionadas con salud oral y embarazo. Av. Odontoestomatol 2009; 25 (3): 147-154.

\section{INTRODUCCIÓN}

Desde tiempos inmemoriales, el ser humano ha buscado dar explicaciones a fenómenos que ocurren a su alrededor. Dentro de éstos, los procesos vitales, así como el proceso salud enfermedad son, por cierto, acontecimientos que especialmente requieren respuestas. La población general, muchas veces explica patologías por creencias basadas en la cultura popular, las que son transmitidas culturalmente a través de generaciones. Dentro de estas se asume, por ejemplo, que el embarazo es causante de una serie de fenómenos en las mujeres, algunos de los cuales se perpetuarían, incluso, una vez que la gravidez ha concluido. Es así como existen creencias culturalmente construidas en torno al embarazo y la salud oral. La creencia que, debido a que los requerimientos de calcio del ser en formación en el vientre materno, las mujeres desarrollarían caries dentales, se encuentra arraigada en diversas culturas (1). Del mismo modo, algunas pacientes señalan y aceptan como proceso normal en el embarazo que "por cada hijo se pierde un diente" (2). Por otro lado, existe la creencia que las mujeres embarazadas no deben recibir atención odontológica por el posible daño que puede producir al embrión o feto el uso de anestésicos locales (2). Lo anterior cobra aun más importan- cia ante el antecedente que existen médicos quienes carecen de información en cuanto al bajo riesgo que poseen los procedimientos odontológicos, tanto terapéuticos como diagnósticos, hecho que se manifiesta en el temor por parte de algunos clínicos en someter a sus pacientes embarazadas a tratamientos dentales (1).

Sin embargo, la evidencia señala que los tejidos dentarios no responden a los cambios bioquímicos del embarazo, así como tampoco el intercambio de calcio es causante del desarrollo de caries dentales (2). Por otro lado, y como producto de los cambios hormonales de las embarazadas, es posible observar una mayor frecuencia de lesiones de tejidos blandos, tales como, gingivitis gravídica con prevalencia desde un 25 a $100 \%$ en las poblaciones estudiadas (2-4), úlceras inespecíficas (4), granuloma piógeno y glositis migratoria benigna (5), sin embargo, estas alteraciones corresponden a un proceso transitorio, que culmina con el fin del embarazo (2).

Un tratamiento odontológico correctamente planificado, no sería causante de alteración del niño en formación (1), por el contrario, existe evidencia que señala que no tratar la enfermedad periodontal durante el embarazo, podría causar partos prematuros 
y bajo peso al nacer, situación que es un problema de salud pública tanto para países desarrollados como para aquellos en desarrollo $(6,7)$. Este hecho cobra mayor importancia cuando se plantea que hasta un $56 \%$ de los médicos no acostumbra a examinar la cavidad oral de sus pacientes (1), lo cual dificulta la observación y tratamiento oportuno de patologías orales que efectivamente pueden complicar un embarazo por infección secundaria no oral a partir de un foco primario oral (8).

Dado el impacto de este tipo de patologías y la complejidad de su investigación resulta de gran importancia la contribución activa de los pacientes en su detección precoz. La posibilidad de un proceso colaborador entre pacientes y personal de salud es sólo posible si se incorporan los elementos culturales, la estructura social, y la historia del grupo objeto de la intervención. En este contexto, la cultura se relaciona profundamente con conceptos como estilo de vida, formas de sociabilización y autoayuda, mecanismos de intervención que serían los cimientos en que se basa la promoción en salud (2).

Una de las estrategias propuestas por la Organización Mundial de la Salud es la Promoción de la Salud, que se define como el proceso que permite a las personas incrementar el control sobre su salud para mejorarla. Este proceso deberá basarse en el conocimiento previo tanto de pautas culturales como de estilos de vida de la población. El no considerar éstos tópicos podría resultar en contenidos culturalmente irrelevantes afectando las posibilidades de éxito del programa aplicado (1).

El objetivo de la investigación ha sido explorar la opinión en torno a la influencia de patologías orales, y la atención odontológica, sobre el curso y término del embarazo en un grupo de embarazadas en control prenatal en centros de salud de la ciudad de Temuco, Chile.

\section{MATERIAL Y MÉTODOS}

Ésta es una investigación exploratoria, transversal, realizada durante el período comprendido entre Agosto y Diciembre del 2007, en 9 centros de salud de la comuna de Temuco, Chile. Las participantes corres- ponden a 65 embarazadas en control prenatal, seleccionadas por conveniencia de acuerdo a los siguientes criterios:

a. Ser mayor de 18 años de edad o contar con la autorización del padre, madre o tutor legal.

b. Encontrarse en control prenatal en centros de salud familiar, consultorios u hospitales públicos de la IX Región de la Araucanía, Chile, adscritos a convenio con la Universidad de La Frontera.

c. Aceptar voluntariamente participar en la investigación por medio de la firma de un consentimiento informado.

La recolección de datos se realizó a través de una encuesta que contempla tres grandes temas: creencias sobre embarazo y salud oral (Tema 1), temores sobre la atención odontológica (Tema 2), y presencia de síntomas y signos auto referidos de patologías orales (Tema 3). La encuesta fue conducida por estudiantes de sexto año de la Carrera de Odontología de la Universidad de La Frontera.

Los datos fueron tabulados e ingresados al programa STATA ${ }^{\circledR}$ 9.0. para realizar cálculo de proporciones, frecuencias, medidas de asociación y tablas de resúmenes.

\section{RESULTADOS}

Las personas que participaron de la investigación poseían, en promedio, 21,46 años de edad, 11,7 años de escolaridad y 22,6 semanas de gestación. Se observó un mayor número de personas de origen urbano, con un $69,23 \%$ de las participantes de la investigación (45 individuos) en comparación a sólo un $30,77 \%$ que procedía de zonas rurales (20 personas). Del total de la muestra, 20 encuestadas declaró ser de origen mapuche $(30,77 \%)$ y $45(69,23 \%)$ declaró no pertenecer a la etnia señalada.

Sobre el total de personas encuestadas, el $95 \%$ cree que durante el embarazo es necesario consultar al odontólogo, el $60 \%$ cree que el embarazo puede producir caries, $70 \%$ de las personas que participó del estudio cree que el embarazo puede producir caída de piezas dentarias, un $75 \%$ ha evidenciado sangrado de encías durante el embarazo, y sólo un $22 \%$ del 
total de personas encuestadas siente temor de consultar al dentista durante el embarazo. El $65 \%$ de las personas de origen Mapuche ha evidenciado sangrado de encías durante el embarazo (Tabla 1).

En la Tabla 2 se muestran los resultados a las opiniones y creencias de las participantes en la investigación según etnia a la que declaran pertenecer (mapuche o no mapuche). Las personas de origen mapuche han señalado en un 95\% que durante el embarazo es necesario consultar al odontólogo, un $55,56 \%$ de ellas asegura que el embarazo puede producir caries. Un 61,11\% de las embarazadas, pertenecientes a la etnia señalada, cree que el embarazo puede producir caída de piezas dentarias y sólo el $15 \%$ de las mujeres mapuches siente temor de consultar al dentista durante el embarazo. Detalle de estos resultados se encuentra en la Tabla 2.
Fecha:

ENCUESTA DE PERCEPCIÓN DE SALUD ORAL DE LA EMBARAZADA PRIMIGESTA

Antecedentes Generales

\begin{tabular}{|l|l|l|l|l|l|l|c|}
\hline \multirow{2}{*}{ Consultorio } & \multirow{2}{*}{$\begin{array}{c}\text { Edad } \\
\text { embarazada }\end{array}$} & \multirow{2}{*}{$\begin{array}{l}\text { Edad (sem) } \\
\text { gestacional }\end{array}$} & \multicolumn{2}{|c|}{ Procedencla } & \multicolumn{2}{|c|}{ Etnla } & Escolaridad \\
\cline { 6 - 7 } & & & Urb & Rur & Map & No Map & (años) \\
\hline & & & & & & & \\
\hline
\end{tabular}

La presente encuesta busca conocer las ideas que ud. posee en relación a la salud bucal durante el embarazo. Solicitamos su colaboración contestando estas preguntas

1.- Hay personas que piensan que las embarazadas no deberían ir al dentista ¿Esta usted de acuerdo con esa opinión?

-Si No

2.- ¿Cree ud. que el embarazo produce o caries (o picaduras)?

$\mathrm{Si}$ No

3.- ¿Piensa ud. que su embarazo puede producir que se le caigan los dientes? Si _ No

4.- ¿Le han sangrado las encías durante el embarazo?

Si No

5.- ¿Tiene miedo de ir al dentista?

Si _ No

Muchas gracias por su colaboración
Anexo 1. Encuesta de salud oral aplicada a embarazadas 
TABLA 1.- CREENCIAS Y OPINIONES DE EMBARAZADAS SOBRE TOTAL DE LA MUESTRA

\begin{tabular}{|c|c|c|c|c|}
\hline \multirow{3}{*}{ RESPUESTAS } & \multicolumn{4}{|c|}{ PREGUNTAS } \\
\hline & \multicolumn{2}{|c|}{$\mathrm{Si}$} & \multicolumn{2}{|c|}{ No } \\
\hline & $\mathbf{n}$ & $\%$ & $\mathbf{n}$ & $\%$ \\
\hline $\begin{array}{l}\text { Las embarazadas no deberían ir al dentista ¿Está usted } \\
\text { de acuerdo con esa opinión? }\end{array}$ & 3 & 4,61 & 62 & 95,39 \\
\hline ¿Cree ud. que el embarazo produce caries (o picaduras)? & 38 & 60,31 & 25 & 39,69 \\
\hline $\begin{array}{l}\text { ¿Piensa ud. que su embarazo puede producir que se le } \\
\text { caigan los dientes? }\end{array}$ & 44 & 70,96 & 18 & 29,04 \\
\hline $\begin{array}{l}\text { ¿Cree ud. que el embarazo puede producir sangrado de } \\
\text { las encías? }\end{array}$ & 49 & 75,38 & 16 & 24,62 \\
\hline ¿Tiene miedo de asistir al dentista durante el embarazo? & 18 & 22,95 & 47 & 77,05 \\
\hline
\end{tabular}

\begin{tabular}{|c|c|c|c|c|c|c|c|}
\hline \multicolumn{8}{|c|}{ TABLA 2.- OPINIONES Y CREENCIAS DE EMBARAZADAS SEGÚN ETNIA } \\
\hline \multirow{3}{*}{ TEMAS } & \multirow{3}{*}{ OPINIONES Y/O CREENCIAS } & & \multicolumn{4}{|c|}{ EtNia } & \multirow{3}{*}{$\mathbf{p}$} \\
\hline & & & \multicolumn{2}{|c|}{ No mapuche } & \multicolumn{2}{|c|}{ Mapuche } & \\
\hline & & & $\mathrm{N}$ & $\%$ & $\mathbf{n}$ & $\%$ & \\
\hline \multirow{3}{*}{1} & Embarazadas no deben ir al dentista & $\begin{array}{c}\mathrm{Si} \\
\mathrm{No}\end{array}$ & $\begin{array}{r}2 \\
43\end{array}$ & $\begin{array}{r}4,44 \\
95,56\end{array}$ & $\begin{array}{r}1 \\
19\end{array}$ & $\begin{array}{r}5,00 \\
95,00\end{array}$ & 1,00 \\
\hline & ¿Embarazo produce caries? & $\begin{array}{l}\mathrm{Si} \\
\text { No }\end{array}$ & $\begin{array}{l}28 \\
17\end{array}$ & $\begin{array}{l}62,22 \\
37,78\end{array}$ & $\begin{array}{r}10 \\
8\end{array}$ & $\begin{array}{l}55,56 \\
44,44\end{array}$ & 0,777 \\
\hline & ¿Embarazo produce caída de dientes? & $\begin{array}{l}\mathrm{Si} \\
\text { No }\end{array}$ & $\begin{array}{l}33 \\
11\end{array}$ & $\begin{array}{l}75,00 \\
25,00\end{array}$ & $\begin{array}{r}11 \\
7\end{array}$ & $\begin{array}{l}61,11 \\
38,89\end{array}$ & 0,357 \\
\hline 2 & ¿Han sangrado sus encías en embarazo? & $\begin{array}{l}\mathrm{Si} \\
\text { No }\end{array}$ & $\begin{array}{r}36 \\
9\end{array}$ & $\begin{array}{l}80,00 \\
20,00\end{array}$ & $\begin{array}{r}13 \\
7\end{array}$ & $\begin{array}{l}65,00 \\
35,00\end{array}$ & 0,223 \\
\hline 3 & ¿Siente temor de ir al dentista? & $\begin{array}{l}\mathrm{Si} \\
\text { No }\end{array}$ & $\begin{array}{l}15 \\
30\end{array}$ & $\begin{array}{l}33,33 \\
66,67\end{array}$ & $\begin{array}{r}3 \\
17\end{array}$ & $\begin{array}{l}15,00 \\
85,00\end{array}$ & 0,148 \\
\hline
\end{tabular}

La Tabla 3 detalla las opiniones y creencias en relación a salud oral y embarazo según origen (área donde habitan, urbano o rural). De las personas que habitan dentro del radio urbano, $95 \%$ de ellas cree que durante el embarazo es necesario consultar al odontólogo; $50 \%$ de las encuestadas de sectores urbanos cree que el embarazo puede producir caries.
El 72,22\% de las personas que viven en el señalado sector cree que el embarazo puede producir caída de piezas dentarias; del mismo modo, $70 \%$ de estas mujeres han evidenciado sangrado de encías durante el embarazo, y finalmente, sólo un el $25 \%$ de las encuestadas con residencia en la zona antes enunciada siente temor de consultar al Dentista Durante 


\section{TABLA 3.- OPINIONES Y CREENCIAS DE EMBARAZADAS SEGÚN LUGAR DE RESIDENCIA}

\begin{tabular}{|c|c|c|c|c|c|c|c|}
\hline \multirow{3}{*}{ TEMAS } & \multirow{3}{*}{\multicolumn{2}{|c|}{ OPINIONES Y/O CREENCIAS }} & \multicolumn{4}{|c|}{ LUGAR DE RESIDENCIA } & \multirow{3}{*}{$\mathrm{p}$} \\
\hline & & & \multicolumn{2}{|c|}{ Urbano } & \multicolumn{2}{|c|}{ Rural } & \\
\hline & & & $\mathbf{N}$ & $\%$ & $\mathbf{n}$ & $\%$ & \\
\hline \multirow{3}{*}{1} & Embarazadas no deben ir al dentista & $\begin{array}{c}\mathrm{Si} \\
\mathrm{No}\end{array}$ & $\begin{array}{r}2 \\
43\end{array}$ & $\begin{array}{r}4,44 \\
95,56\end{array}$ & $\begin{array}{r}1 \\
19\end{array}$ & $\begin{array}{r}5,00 \\
95,00\end{array}$ & 1,00 \\
\hline & ¿Embarazo produce caries? & $\begin{array}{l}\mathrm{Si} \\
\text { No }\end{array}$ & $\begin{array}{l}28 \\
15\end{array}$ & $\begin{array}{l}65,12 \\
34,88\end{array}$ & $\begin{array}{l}10 \\
10\end{array}$ & $\begin{array}{l}50,00 \\
50,00\end{array}$ & 0,281 \\
\hline & ¿Embarazo produce caída de dientes? & $\begin{array}{c}\mathrm{Si} \\
\text { No }\end{array}$ & $\begin{array}{l}13 \\
31\end{array}$ & $\begin{array}{l}29,55 \\
70,45\end{array}$ & $\begin{array}{r}13 \\
5\end{array}$ & $\begin{array}{l}72,22 \\
27,78\end{array}$ & 1,00 \\
\hline 2 & ¿Han sangrado sus encías en embarazo? & $\begin{array}{c}\mathrm{Si} \\
\text { No }\end{array}$ & $\begin{array}{l}35 \\
10\end{array}$ & $\begin{array}{l}77,78 \\
22,22\end{array}$ & $\begin{array}{r}14 \\
6\end{array}$ & $\begin{array}{l}70,00 \\
30,00\end{array}$ & 0,542 \\
\hline 3 & ¿Siente temor de ir al dentista? & $\begin{array}{c}\mathrm{Si} \\
\mathrm{No}\end{array}$ & $\begin{array}{l}13 \\
32\end{array}$ & $\begin{array}{l}28,89 \\
71,11\end{array}$ & $\begin{array}{r}5 \\
15\end{array}$ & $\begin{array}{l}25,00 \\
75,00\end{array}$ & 1,00 \\
\hline
\end{tabular}

el embarazo. Detalles de los resultados según origen en la Tabla 3.

\section{DISCUSIÓN}

Aunque existe documentación que las embarazadas rechazan la atención odontológica producto del potencial daño de este procedimiento al bebé en desarrollo (2), los resultados de esta investigación contradicen esos supuestos, ya que la gran mayoría de las encuestadas no está de acuerdo con quienes piensan que durante el embarazo no deberían asistir al dentista. Esta tendencia se mantuvo según etnia, donde ambos grupos manifestaron ampliamente estar en desacuerdo con esta afirmación ( $p>0,05)$, repitiéndose esto según lugar de residencia $(p>0,05)$.

A pesar de la evidencia que el embarazo per se no es factor causal del desarrollo de caries dentales (2), existe la creencia entre las participantes de este estudio que si las produce. Esta afirmación es observada frecuentemente en pacientes mapuches $(p>0,05)$ y en personas de procedencia rural $(p>0,05)$.

Grabero y cols. (2) han señalado que las personas creen que durante el embarazo es esperable la pérdi- da de piezas dentales. Esta situación también ha sido manifestada por las mujeres de este estudio, quienes en su mayoría piensan que esta es una situación posible de acaecer durante el embarazo, especialmente las mujeres mapuches ( $p>0,05)$; y mujeres de origen rural $(p>0,05)$. Los dos puntos antes desarrollados señalan la existencia de una tarea pendiente en el personal de salud que trabaja directamente con mujeres embarazadas, relacionada principalmente con la educación de este grupo de personas a este respecto, ya que en las encuestadas prevalece una creencia, probablemente traspasada generacionalmente, sobre hechos científicamente comprobados. Conocer los hechos señalados podría motivar a las futuras madres en desarrollar medidas de autocuidado en salud oral, bajo la premisa de modificar acontecimientos atribuidos inherentemente a la condición de gravidez, por situaciones que corresponden a enfermedades posibles de prevenir.

Según Grabero y cols. (2), Offenbacher y cols. (3), Díaz y cols. (5), una de las patologías de tejidos blandos más observada durante el embarazo es la gingivitis gravídica el cual es un proceso de carácter transitorio, que culmina con el fin del embarazo (2). Esta alteración se evidencia mediante auto exa- 
men, y a través de la observación de sangrado de encías. Este estudio no contempló un análisis clínico de las pacientes encuestadas, por lo que no es posible establecer una correlación positiva entre lo que las pacientes declaran y la prevalencia de la patología en esta población. No obstante, un número importante de personas señaló presentar hemorragia gingival, de los cuales son en su mayoría no mapuches y de residencia urbana. Dado que el auto reporte puede ser considerado una información certera, lo anterior apunta a la posibilidad de encontrar clínicamente patología gingival en una cantidad importante de las pacientes embarazadas con características similares. Ante esta situación, y considerando la posibilidad de prevenir la gingivitis con medidas relativamente sencillas de autocuidado, surge una vez más, la necesidad de reforzar las acciones tendentes a promover la salud oral en este grupo de personas, con el ánimo de conservar y mantener tanto la salud oral de las personas participantes de este estudio, como la de sus hijos por nacer.

En relación a los temores de asistir al dentista, y a pesar de ser una creencia ampliamente difundida incluso en algún sector del personal de salud $(1,2)$ sólo un $27 \%$ declaró sentir miedo ante la posibilidad de recibir atención odontológica, con más temor en personas no mapuches comparadas con personas mapuches $(>0,05)$, y en personas de residencia en áreas urbanas comparadas con personas habitantes de zonas rurales $(p>0,05)$. Este dato resulta interesante, ya que podría señalar la existencia de una cantidad importante de embarazadas dispuestas a participar de programas de intervención de salud oral transversales a todos los niveles de prevención, incluso en los aspectos de limitación del daño y rehabilitación, aspectos en que, según la literatura consultada, existiría reticencia a hacer participar a sus pacientes a algún sector de médicos tratantes de mujeres embarazadas (1).

Es posible señalar entonces que se requieren mayores esfuerzos de todo el personal de salud en educación durante el control prenatal para entregar conocimientos adecuados y correctos a las embarazadas en relación a salud oral y embarazo, que permita mejorar las medidas de auto cuidado, y por lo tanto, la salud oral de estas personas durante éste período, y eventualmente, el resto de sus vidas, lo que podría perpetuarse incluso es sus hijos por nacer.

Lo anteriormente señalado debe planificarse considerando la influencia de las creencias culturales sobre el comportamiento en salud de las personas. Los datos expuestos en este estudio proporcionan al personal de salud que interactúa con el binomio madre-hijo desde antes del nacimiento, información relevante para generar procesos de educación y promoción de la salud oral de las madres y de las futuras generaciones. No considerar las variables socioculturales en la planificación de estrategias de promoción, impediría lograr un impacto significativo en la salud de las personas, y a la vez disminuiría el éxito de los programas de prevención junto con desaprovechar los recursos técnicos y humanos destinados a la educación en salud de las embarazadas.

\section{BIBLIOGRAFÍA}

1. Díaz-Romero R, Martínez-Sánchez C, Vertiz-Ramírez, Rodríguez-Saldívar M. Mitos, prejuicios y realidades del tratamiento odontológico durante el embarazo. Opinión Médica. Ginec Obst Mex 1998;66:507.

2. Garbero I, Delgado A, Benito de Cárdenas I. Salud oral en embarazadas: Conocimientos y Actitudes. Acta odontológica Venezolana 2005; (43)2.

3. Offenbacher S, Boggess K, Murtha A P, Jared H L, Leiff S, Mc Kaig R., Mauriello S, Moss K L, Beck J. Progressive Periodontal Disease and Risk of Very Preterm Delivery. Obstet Gynecol 2006; 107:29-36.

4. Lárez L, Benavides Y, Henríquez Y, Moreno S. Lesiones Bucales vistas en la Embarazada. Rev Obstet Ginecol Venez 2005;(65)1.

5. Díaz L, Castellanos J. Lesiones de la Mucosa bucal y comportamiento de la enfermedad periodontal en embarazadas. Med. Oral Patol. Oral cir. Bucal 2004;(9)5. 
6. Urban E, Radnai M, Novák T, Gorzó I, Pál A, Nagy E. Distribution of anaerobic bacteria among pregnant periodontitis patients who experience preterm delivery. Anaerobe 2006;12:52-7.

7. Yiping H, Ikegami A, Bissada N, Herbst M, Redline $\mathrm{R}$, Ashmead G. Transmission of an uncultived Bergeyrella Strain from the oral cavity to amniotic fluid in a case of preterm birth. J Clin Microbiol Apr 2006, p. 1475-83.

8. Xiaoling LI, Kolltveit K, Tronstad L, Olsen I. Systemic Diseases caused by oral infection.
Clinical Microbiology Reviews, Oct. 2000 p. 54758.

\section{CORRESPONDENCIA}

Prof. Ramón Fuentes Fernández

Profesor asociado Departamento de Odontología Integral

Facultad de Medicina. Universidad de La Frontera Manuel Montt, $112,4^{\circ}$ Piso

Temuco, Chile.

E-mail: rfuentes@ufro.cl 